\title{
DETERMINACIÓN DE LA EFICIENCIA DE LA MICROPROPAGACIÓN DE GENOTIPOS DE MANDIOCA (MANIHOT ESCULENTA, EUPHORBIACEAE) DE interés para el Nordeste Argentino
}

\author{
RICARDO DANIEL MEDINA, SILVIA CRISTINA SCHALLER ${ }^{1 *}$, NATALIA RAQUEL DOLCE y \\ LUIS AMADO MROGINSKI ${ }^{2}$
}

\begin{abstract}
Summary: Micropropagation efficiency determination of cassava (Manihot esculenta, Euphorbiaceae) genotypes of interest to the Northeast of Argentina. In vitro cassava propagation has been reported in numerous studies; however, the efficiency of multiplication had only been established in a very restricted number of cultivars and / or subcultures during micropropagation. In order to evaluate the in vitro multiplication of 20 economically important cassava cultivars of Northeast Argentina (six total subcultures performed every 30 days), uninodal segments were cultured in basal Murashige and Skoog (1962) supplemented with $0.01 \mathrm{mg} / \mathrm{L}$ of 1 -naphthaleneacetic acid, $0.01 \mathrm{mg} / \mathrm{L}$ of 6 -benzylaminopurine and 0.1 $\mathrm{mg} / \mathrm{L}$ gibberellic acid. Plant regeneration was possible in $100 \%$ of the cultivars during the 6 subcultures. Regardless of the subculture, the average number of nodes regenerated per explant varied significantly among cultivar from two to five nodes per plants $(P \leq 0,0001)$. The average number of accumulated nodes was significantly different among cultivars with values that ranged from 46 to 16,568 nodes per plants $(P \leq 0,0001)$. Results show variability due to genotype, distinguishing three cultivars groups according to their propagation efficiency and growth in length ability. Determining the multiplication rate of different cassava cultivars is essential for optimizing micropropagation.
\end{abstract}

Key words: Genotype effect, micropropagation, subcultures, multiplication rate, cassava.

Resumen: En la bibliografía se citan numerosos trabajos de propagación in vitro de mandioca, sin embargo sólo se ha establecido la eficiencia de la multiplicación para un número muy restringido de cultivares y/o subcultivos durante la micropropagación. El objetivo de este trabajo fue evaluar la multiplicación in vitro de 20 cultivares de mandioca de interés económico para el Nordeste Argentino (6 subcultivos totales realizados cada 30 días). Para ello, se cultivaron segmentos uninodales en medio basal de Murashige y Skoog (1962) más 0,01 mg/L de ácido 1-naftalenacético, 0,01 mg/L de 6-bencilaminopurina y $0,1 \mathrm{mg} / \mathrm{L}$ de ácido giberélico. Todos los cultivares evaluados regeneraron plantas durante los 6 subcultivos. Independientemente del subcultivo, el número promedio de nudos regenerados por explante varió significativamente con el cultivar $(P \leq 0,0001)$, rondando valores de 2 a 5 nudos por planta. El número promedio de nudos acumulados fue significativamente distinto dependiendo del cultivar, alcanzando valores de 46 a 16.568 nudos totales por plantas $(P \leq 0,0001)$. Los resultados demuestran variabilidad debida al genotipo, distinguiéndose 3 grupos de cultivares en relación a su capacidad de propagación y crecimiento en longitud. La determinación de la tasa de multiplicación de distintos cultivares de mandioca es fundamental para la optimización de la micropropagación.

Palabras clave: efecto del genotipo, micropropagación, subcultivos, tasa de multiplicación, mandioca.

\footnotetext{
$1 *$ ex aequo

${ }^{2}$ Instituto de Botánica del Nordeste (Universidad Nacional del Nordeste - Consejo Nacional de Investigaciones Científicas y Técnicas). Facultad de Ciencias Agrarias, Universidad Nacional del Nordeste. Sargento Cabral 2131, CC. 209 (3400), Corrientes, Argentina. E-mail: ricardomedina@agr.unne.edu.ar
} 


\section{INTRODUCCIÓN}

La mandioca (Manihot esculenta Crantz) es uno de los principales cultivos a nivel mundial y constituye una de las fuentes de hidratos de carbono más utilizadas en la alimentación y en la industria (Cock, 1985). Esta especie es diclino monoica y de polinización cruzada, por lo que cada individuo proveniente de semilla presenta un alto grado de heterocigosis (Ceballos y de la Cruz, 2002). Ésta es una de las razones por las que su cultivo comercial es agámico, mediante la plantación de estacas caulinares (El-Sharkawy, 2003). Un inconveniente de la propagación vegetativa convencional es que facilita la rápida diseminación de plagas y enfermedades, afectando al material de plantación (Puonti-Kaerlas, 1998). A esto se suma la baja tasa de multiplicación (López, 2002; Demeke et al., 2014), ya que una planta madura sólo es capaz de brindar 10 a 20 estacas por año (Smith et al., 1986), dificultando la multiplicación y la difusión de nuevos cultivares. En este contexto, la biotecnología puede contribuir en la solución de estos problemas (Fregene et al., 2002), brindando como alternativa la posibilidad de propagar masivamente plantas sanas mediante el cultivo in vitro.

En la bibliografía se describieron numerosos métodos de propagación in vitro de mandioca basados en la vía organogénica (Roca, 1984; Smith et al., 1986; Roca et al., 1991; Mussio et al., 1998; Albarrán et al., 2003; Hankoua et al., 2005; Cavallero et al., 2012). Sin embargo, en general se ha tratado superficialmente su eficiencia y en los casos en que la tasa de multiplicación se ha calculado se restringe a un escaso número de cultivares (Pedroso de Oliveira et al., 2000; Acedo, 2006) y subcultivos durante el cultivo in vitro (Acedo, 1994; Bromees y Lacon, 1994). Es importante entonces evaluar el comportamiento in vitro de un conjunto diverso de cultivares de mandioca durante subcultivos sucesivos y de esta manera poder establecer una tasa de multiplicación representativa para los materiales más promisorios.

El objetivo de este trabajo fue evaluar el comportamiento in vitro de segmentos uninodales de 20 cultivares de mandioca durante 6 subcultivos para calcular la tasa de multiplicación, una herramienta logística indispensable para el establecimiento de un programa de propagación masal de esta especie.

\section{Materiales y Métodos}

\section{Material vegetal}

En este estudio se evaluaron 20 cultivares de mandioca (Manihot esculenta) de interés económico para el Nordeste Argentino (NEA) provenientes de: Cuba (cv. Cuba Señorita), Colombia (cvs. MCol 1505, CM 3372-4 y CM 3306-4 del Centro Internacional de Agricultura Tropical), Brasil (cvs. IAC 12.829 del Instituto Agronômico de Campinas y Surubim-41 de la Empresa Brasileira de Pesquisa Agropecuária, Recursos Genéticos e Biotecnologia), Paraguay (cv. MPar 75) y recolectados en Argentina por personal de la Facultad de Ciencias Agrarias de la Universidad Nacional del Nordeste (cvs. Amarilla, Carapé y Catiguá) y por investigadores del Instituto Nacional de Tecnología Agropecuaria, Estación Experimental Agropecuaria El Colorado (EC), Formosa (EC 11, EC 27, EC 55, EC 64, EC 78, EC 118, 9, 60, 76 y 1468). Los mismos se preservan en el Banco de Germoplasma que se mantiene en condiciones in vitro en el Laboratorio de Cultivo de Tejidos Vegetales (Instituto de Botánica del Nordeste -Universidad Nacional del Nordeste Consejo Nacional de Investigaciones Científicas y Técnicas- y Facultad de Ciencias Agrarias, Universidad Nacional del Nordeste) utilizando el procedimiento descripto por Caballero et al. (2012). Dicho protocolo consiste en el cultivo de segmentos nodales en el medio de micropropagación compuesto por el medio basal sugerido por Murashige y Skoog (1962) -MS-, con 3\% de sacarosa, suplementado con $0,01 \mathrm{mg} / \mathrm{L}$ de ácido naftalenacético (ANA), 0,01 $\mathrm{mg} / \mathrm{L}$ de 6 -bencilaminopurina (BAP) y $0,1 \mathrm{mg} / \mathrm{L}$ de ácido giberélico $\left(\mathrm{AG}_{3}\right)$.

\section{Micropropagación}

Se evaluó la eficiencia de la micropropagación a partir del cultivo de segmentos uninodales basales de las plantas regeneradas in vitro, subcultivándolos cada 30 días durante 180 días, alcanzando un total de 6 ciclos de subcultivos en el medio descripto en Cavallero et al., (2012). El pH de los medios de cultivo fue ajustado a 5,8 con soluciones de $\mathrm{KOH}$ y/o $\mathrm{HCl}$ antes del agregado de $0,75 \%$ de agar (A-1296, Sigma-Aldrich). Los medios fueron esterilizados en autoclave a 1 atmósfera de presión $\left(120^{\circ} \mathrm{C}\right)$ durante 20 min. La incubación se realizó en una cámara climatizada a $27 \pm 2^{\circ} \mathrm{C}, 14 \mathrm{~h}$ de fotoperíodo y una irradiancia de $116 \mu \mathrm{mol} \mathrm{m} \mathrm{m}^{-2} \mathrm{~s}^{-1}$. 


\section{Diseño experimental y análisis estadísticos}

El diseño experimental fue de bloques completos al azar, donde se evaluaron 20 cultivares por 6 subcultivos realizados cada 30 días durante 180 días. El experimento fue repetido 3 veces con 10 explantes por repetición, analizando las respuestas de 30 segmentos uninodales en cada subcultivo $(\mathrm{n}=30)$.

Para determinar la eficiencia de la micropropagación se evaluó i) el número promedio de nudos regenerados por explante por subcultivo cada 30 días, ii) el número promedio de nudos acumulados totales al cabo de 180 días de cultivo y iii) la longitud promedio de los vástagos regenerados por explante por subcultivo cada 30 días.

Los datos fueron sujetos al análisis de la variancia y la comparación de las medias se realizó mediante el test de comparaciones múltiples de Duncan
$(\mathrm{P} \leq 0,05)$. Las relaciones fenotípicas entre cultivares se representaron gráficamente mediante un análisis de conglomerados utilizando el promedio aritmético de grupos de pares no ponderados (UPGMA) y la distancia Euclidiana promedio. El ajuste entre ambas matrices se realizó mediante el coeficiente de correlación cofenética. También se realizó un análisis de variancia multivariado con posterior comparación de medias mediante la prueba de Hotelling $(\mathrm{P} \leq 0,05)$. Todos los análisis se realizaron con el programa InfoStat versión 2008 (Di Rienzo et al., 2008).

\section{Resultados y Discusión}

En la Tabla 1 se presentan los resultados obtenidos respecto al número promedio de

Tabla 1. Efecto del genotipo y el número de subcultivos sobre el número promedio de nudos

regenerados por explante y el número promedio de nudos acumulados totales de mandioca (Manihot esculenta) multiplicados in vitro.

\begin{tabular}{|c|c|c|c|c|c|c|c|c|}
\hline \multirow{2}{*}{ Cultivar } & \multicolumn{7}{|c|}{ Subcultivos } & \multirow{2}{*}{$\begin{array}{l}\mathrm{N}^{0} \text { nudos } \\
\text { acumulados }\end{array}$} \\
\hline & 1 & 2 & 3 & 4 & 5 & 6 & Media & \\
\hline Cuba Señorita & $5,2 \pm 0,2^{* 1}$ & $5,1 \pm 0,3$ & $5,7 \pm 0,4$ & $5,2 \pm 0,2$ & $5,0 \pm 0,5$ & $4,2 \pm 0,1$ & $5,1 a^{* 2}$ & $16.568,4 \mathrm{a}$ \\
\hline MCol 1505 & $4,3 \pm 0,2$ & $5,5 \pm 0,1$ & $4,7 \pm 0,4$ & $5,0 \pm 0,2$ & $5,1 \pm 0,2$ & $3,9 \pm 0,3$ & $4,8 \mathrm{a}$ & $11.166,3 \mathrm{c}$ \\
\hline CM 3372-4 & $5,0 \pm 0,1$ & $4,6 \pm 0,6$ & $5,4 \pm 0,3$ & $5,0 \pm 0,2$ & $4,9 \pm 0,2$ & $4,6 \pm 0,1$ & $4,9 \mathrm{a}$ & $13.732,1 \mathrm{~b}$ \\
\hline 9 & $3,6 \pm 0,2$ & $3,7 \pm 0,3$ & $5,3 \pm 0,1$ & $3,6 \pm 0,3$ & $3,9 \pm 0,2$ & $4,0 \pm 0,2$ & $4,0 \mathrm{~b}$ & $4.190,4 \mathrm{~d}$ \\
\hline Surubim-41 & $5,0 \pm 0,5$ & $3,8 \pm 0,3$ & $4,5 \pm 0,4$ & $3,4 \pm 0,2$ & $3,5 \pm 0,1$ & $2,9 \pm 0,1$ & $3,8 \mathrm{~b}$ & $2.985,4 \mathrm{de}$ \\
\hline IAC 12.829 & $4,0 \pm 0,4$ & $3,3 \pm 0,4$ & $3,3 \pm 0,7$ & $3,8 \pm 0,1$ & $4,8 \pm 0,0$ & $3,9 \pm 0,1$ & $3,8 \mathrm{~b}$ & $3.022,8 \mathrm{de}$ \\
\hline 1468 & $4,3 \pm 0,1$ & $4,5 \pm 0,2$ & $4,3 \pm 0,1$ & $3,6 \pm 0,2$ & $2,7 \pm 0,4$ & $2,7 \pm 0,4$ & $3,7 \mathrm{cb}$ & $2.333,1$ def \\
\hline 76 & $3,7 \pm 0,4$ & $3,9 \pm 0,3$ & $3,7 \pm 0,4$ & $3,6 \pm 0,2$ & $4,0 \pm 0,5$ & $3,1 \pm 0,2$ & $3,7 \mathrm{cb}$ & $2.364,5$ def \\
\hline Amarrilla & $3,8 \pm 0,3$ & $4,1 \pm 0,1$ & $4,1 \pm 0,4$ & $4,7 \pm 0,2$ & $3,3 \pm 0,4$ & $2,8 \pm 0,4$ & $3,7 \mathrm{cb}$ & $2.369,4$ def \\
\hline CM 3360-4 & $4,6 \pm 0,3$ & $3,8 \pm 0,7$ & $3,3 \pm 0,1$ & $3,2 \pm 0,4$ & $3,7 \pm 0,1$ & $3,3 \pm 0,1$ & $3,7 \mathrm{cb}$ & $2.202,2$ def \\
\hline 60 & $4,4 \pm 0,2$ & $4,0 \pm 0,2$ & $3,6 \pm 0,1$ & $3,8 \pm 0,1$ & $3,2 \pm 0,2$ & $1,3 \pm 0,1$ & $3,4 \mathrm{dc}$ & $1.077,7$ ef \\
\hline Catiguá & $3,8 \pm 0,4$ & $4,1 \pm 0,4$ & $3,4 \pm 0,1$ & $2,9 \pm 0,4$ & $2,9 \pm 0,1$ & $2,2 \pm 0,1$ & $3,2 \mathrm{~d}$ & 935,7 ef \\
\hline M Par 75 & $4,3 \pm 0,1$ & $3,8 \pm 0,4$ & $2,3 \pm 0,2$ & $2,6 \pm 0,3$ & $2,8 \pm 0,1$ & $3,3 \pm 0,2$ & $3,2 \mathrm{~d}$ & 904,2 ef \\
\hline EC 55 & $4,1 \pm 0,1$ & $3,6 \pm 0,3$ & $3,3 \pm 0,5$ & $2,9 \pm 0,2$ & $2,5 \pm 0,1$ & $2,3 \pm 0,1$ & $3,1 \mathrm{~d}$ & 842,2 ef \\
\hline Carapé & $3,7 \pm 0,1$ & $3,5 \pm 0,1$ & $2,7 \pm 0,5$ & $2,3 \pm 0,2$ & $1,2 \pm 0,1$ & $1,4 \pm 0,3$ & $2,5 d$ & $124,4 \mathrm{f}$ \\
\hline EC 11 & $3,1 \pm 0,2$ & $2,7 \pm 0,2$ & $2,5 \pm 0,3$ & $3,3 \pm 0,3$ & $2,2 \pm 0,4$ & $2,6 \pm 0,2$ & $2,7 \mathrm{e}$ & 378,9 ef \\
\hline EC 27 & $3,5 \pm 0,3$ & $3,1 \pm 0,1$ & $3,2 \pm 0,2$ & $2,1 \pm 0,2$ & $2,3 \pm 0,1$ & $1,8 \pm 0,2$ & $2,7 \mathrm{e}$ & 304,8 ef \\
\hline EC 118 & $3,0 \pm 0,2$ & $3,2 \pm 0,3$ & $2,2 \pm 0,2$ & $2,7 \pm 0,2$ & $1,9 \pm 0,1$ & $1,9 \pm 0,2$ & $2,5 \mathrm{fe}$ & 213,9 ef \\
\hline EC 64 & $2,9 \pm 0,2$ & $3,0 \pm 0,2$ & $1,7 \pm 0,3$ & $1,8 \pm 0,1$ & $1,4 \pm 0,2$ & $2,2 \pm 0,1$ & $2,2 \mathrm{gf}$ & $65,7 f$ \\
\hline EC 78 & $2,7 \pm 0,3$ & $2,1 \pm 0,2$ & $2,1 \pm 0,2$ & $2,3 \pm 0,3$ & $1,2 \pm 0,2$ & $1,5 \pm 0,1$ & $1,9 \mathrm{~g}$ & $46,0 \mathrm{f}$ \\
\hline $\begin{array}{l}\text { Media por } \\
\text { subcultivo }\end{array}$ & $3,9 \mathrm{a}$ & $3,8 \mathrm{~b}$ & $3,6 \mathrm{c}$ & $3,4 d$ & $3,1 \mathrm{e}$ & $2,8 \mathrm{f}$ & $3,43 \pm 0,2$ & $3.291,4 \pm 1.066,3$ \\
\hline CV (\%) & 17,7 & 21,0 & 32,3 & 28,8 & 39,7 & 35,2 & 25,3 & --- \\
\hline
\end{tabular}

\footnotetext{
${ }^{* 1}$ Media aritmética \pm error estándar.
}

*2 Letras distintas indican diferencias significativas según el test de comparaciones múltiples de Duncan $(\mathrm{P} \leq 0,05)$. 
nudos regenerados por explante y al número promedio de nudos acumulados al cabo de los 6 ciclos de cultivo realizados, en los 20 cultivares de mandioca evaluados. En el 100\% de los cultivares se regeneraron plantas durante la fase de multiplicación in vitro que comprendió un total de 180 días, aunque con una eficiencia dependiente del genotipo.

Como se puede observar en la Tabla 1, el número promedio de nudos regenerados por explante o tasa de multiplicación varió significativamente con el genotipo $(\mathrm{P} \leq 0,0001)$ alcanzando valores promedio de 1,9 en el cv. EC 78 y 5,1 nudos en el cv. Cuba Señorita. También el número de subcultivos fue una fuente de variación significativa del número de nudos regenerados por explante $(\mathrm{P} \leq 0,0001)$, registrándose el máximo valor en el primer subcultivo $(3,9)$ y disminuyendo progresivamente hasta arrojar valores significativamente más bajos en el sexto subcultivo $(2,8)$. La interacción de los factores principales (genotipo versus subcultivos) demostró ser altamente significativa $(\mathrm{P} \leq 0,0001)$.

En el presente estudio, para el cálculo de la tasa de multiplicación se consideraron los resultados obtenidos sólo en la fase homónima. No se incluyeron los datos obtenidos en la fase de establecimiento in vitro, en donde el material recién se está adaptando a las condiciones de cultivo y es muy variable en su comportamiento, tal como lo indicaran Acedo (1994) y Broomes y Lacon (1994). Pedroso de Oliveira et al. (2000) informaron una tasa media de multiplicación de 2,9 por subcultivo de 30 días en 6 cultivares provenientes de la región nordeste del Brasil, los cuales fueron cultivados en un medio de cultivo constituido por $35 \%$ de los macro y micronutrientes del MS con $2 \%$ de sacarosa, suplementado con $1 \mathrm{mg} / \mathrm{L}$ de tiamina, $100 \mathrm{mg} / \mathrm{L}$ de inositol, 0,01 $\mathrm{mg} / \mathrm{L}$ de ANA y $0,01 \mathrm{mg} / \mathrm{L}$ de $\mathrm{AG}_{3}$. Anteriormente, Acedo (1994) obtuvo tasas de 4 a 6 nudos por subcultivo de 60 días, es decir una eficiencia de multiplicación mensual comprendida en el rango de valores observado en el presente estudio. Broomes y Lacon (1994) registraron tasas de 2 a 6 nudos por subcultivo usando medios de cultivo diferentes a los empleados en el presente trabajo, recomendando que se realicen modificaciones del contenido de nitrógeno, sacarosa y reguladores de crecimiento para adecuar el medio a los distintos genotipos. Acedo (2006) demostró que para el cultivar de mandioca Golden Yellow el medio de micropropagación utilizado, similar al empleado en el presente trabajo, no resultó muy efectivo por lo que sugirió eliminar los reguladores de crecimiento de su formulación. La tasa de multiplicación de 3 a 4 nudos por explante para el cv. Golden Yellow citada por Acedo (2006), no difirió de los valores reportados en este estudio para la media general encontrada en todos los cultivares. Estos resultados permiten corroborar la reproducibilidad y efectividad del procedimiento empleado incluso para un conjunto diverso de cultivares de mandioca, aunque es necesario optimizar la formulación del medio en el caso de cultivares con baja eficiencia de propagación.

Al cabo de 180 días, el número promedio de nudos acumulados totales fue significativamente distinto dependiendo del cultivar $(\mathrm{P} \leq 0,0001)$, alcanzando un valor medio de 46 en el cv. EC78 y 16.568 en el cv. Cuba Señorita (Tabla 1). Pedroso de Oliveira et al. (2000) también observaron un pronunciado efecto del genotipo en la eficiencia de la micropropagación, con un rango de 55,8 a 535,1 nudos acumulados totales luego de 5 subcultivos de 30 días cada uno. Ese efecto ya había sido descripto tempranamente en mandioca por Roca (1984). Hasta el presente no se ha establecido un único procedimiento de micropropagación que demuestre la misma efectividad para distintos genotipos. En este trabajo se ha obtenido un promedio general mayor a 3.000 nudos acumulados totales al cabo de 180 días de cultivo a partir de un solo segmento uninodal, lo que demuestra la potencialidad el procedimiento empleado.

Para calificar apropiadamente un sistema de micropropagación además de considerar la eficiencia en la formación de nuevos nudos o tasa de multiplicación, es importante evaluar la altura de los vástagos regenerados, la diferenciación de callos en los explantes y las pérdidas ocurridas por contaminación microbiana (Pedroso de Oliveira et $a l ., 2000)$. En este ensayo, la longitud de vástagos regenerados por explante mostró variaciones significativas relacionadas con el genotipo $(\mathrm{P} \leq 0,0001)$, registrándose valores promedio de $1,1 \mathrm{~cm}$ en el cv. EC 78 y de 7,2 cm en el cv. CM 3372-4 (Fig. 1), con una media aritmética de 3,3 $\mathrm{cm}$ para los 20 genotipos evaluados (Tabla 2). Con respecto al efecto del número de subcultivos sobre la longitud de vástagos, a partir del cuarto 


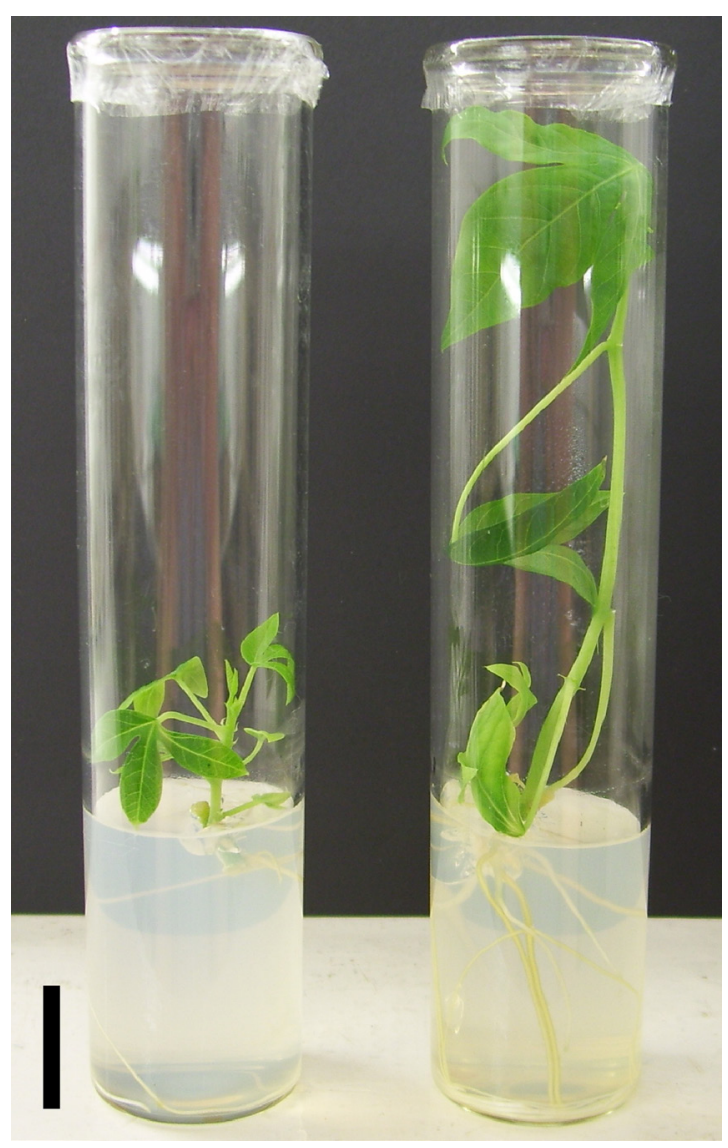

Fig. 1. Efecto del genotipo sobre la longitud de vástagos de mandioca regenerados in vitro. Izquierda: cv. EC78; derecha: cv. CM3372-4. Barra $=1 \mathrm{~cm}$.

subcultivo se observó una disminución en la altura promedio de vástagos, la cual fue de $3 \mathrm{~cm}$. Hacia el sexto subcultivo, la altura de vástagos disminuyó significativamente, alcanzando una media aritmética de $2,3 \mathrm{~cm}$. Existió además una interacción significativa entre los cultivares y los subcultivos en relación a la longitud de vástagos $(\mathrm{P} \leq 0,0001)$. Pedroso de Oliveira et al. (2000) también observaron una gran variación de la longitud de los vástagos regenerados debida al genotipo. Ellos informaron que, en general, fue muy frecuente la regeneración de plantas poco desarrolladas con una altura promedio de apenas $2,4 \mathrm{~cm}$ ( $\sin$ discriminar cultivares ni subcultivos). Sólo uno de los cultivares evaluados presentó una altura promedio de $4,4 \mathrm{~cm}$ que fue el que mostró las mayores alturas a lo largo de los subcultivos y que coincidentemente fue el más prolífico en la regeneración de nudos por explante. Asimismo, Pedroso de Oliveira et al. (2000) evidenciaron que la longitud máxima de vástagos se producía en el cuarto subcultivo para luego decrecer, a diferencia de lo demostrado en este trabajo en donde la longitud decreció progresivamente con los subcultivos. Por otra parte, la sugerencia de Acedo (2006) de no adicionar reguladores de crecimiento vegetal el medio nutritivo para obtener buenos resultados en la propagación del cv. Golden Yellow, es discutible puesto que para hallar la eficiencia sólo consideró un subcultivo y se desconoce si los vástagos podrán elongar adecuadamente en medio libre de reguladores de crecimiento durante repiques sucesivos. En este trabajo, a pesar de que los explantes fueron transferidos mensualmente a medios frescos provistos de reguladores de crecimiento, la altura de los vástagos regenerados disminuyó a medida que aumentó el número de subcultivos.

El fenograma utilizado para representar las relaciones fenotípicas en atributos de eficiencia de multiplicación produjo un buen ajuste con respecto a la matriz de distancia utilizada, siendo el coeficiente de correlación cofenética de 0,731. De acuerdo al fenograma de distancia (Fig. 2), fue posible identificar variabilidad fenotípica y distinguir 3 grupos bien definidos con una línea de corte de 1; uno conformado por los cultivares más proliferativos y que regeneran los vástagos más altos (Cuba Señorita, MCol 1505 y CM 3372-4) y otro constituido por cultivares caracterizados por ser los menos proliferativos y por regenerar vástagos de altura inferior a 2,2 cm (Carapé, EC27, EC11, EC118, EC64 y EC78) (prueba de Hotelling, $\mathrm{P} \leq 0,05$ ). Ubicado entre los grupos extremos, se pudo observar un grupo de cultivares de comportamiento intermedio en donde se agruparon la mayoría de los cultivares analizados (Fig. 2). Evidenciando así la necesidad de optimizar el medio de cultivo a los efectos de mejorar la regeneración de plantas en los cultivares menos proliferativos, como se sugirió en el trabajo de Smith et al. (1986).

Independientemente del genotipo, durante este experimento se observó un desarrollo normal de hojas de las plantas in vitro en cuanto a la 
Tabla 2. Efecto del genotipo y el número de subcultivos sobre la longitud de vástagos regenerados por explante de mandioca (Manihot esculenta) regenerados in vitro.

\begin{tabular}{|c|c|c|c|c|c|c|c|}
\hline \multirow{2}{*}{ Cultivar } & \multicolumn{6}{|c|}{ Subcultivos } & \multirow{2}{*}{$\begin{array}{c}\text { Longitud } \\
\text { media }(\mathrm{cm})\end{array}$} \\
\hline & 1 & 2 & 3 & 4 & 5 & 6 & \\
\hline Cuba Señorita & $6,2 \pm 1,0^{* 1}$ & $6,1 \pm 0,5$ & $7,3 \pm 1,0$ & $6,5 \pm 0,2$ & $6,7 \pm 1,2$ & $4,1 \pm 0,1$ & $6,1 b^{* 2}$ \\
\hline MCol 1505 & $4,6 \pm 1,1$ & $7,4 \pm 0,2$ & $5,9 \pm 1,1$ & $6,8 \pm 0,7$ & $8,0 \pm 0,5$ & $5,5 \pm 0,3$ & $6,4 \mathrm{~b}$ \\
\hline CM 3372-4 & $6,3 \pm 0,3$ & $6,2 \pm 0,3$ & $9,1 \pm 0,7$ & $7,1 \pm 0,7$ & $7,5 \pm 1,0$ & $6,7 \pm 0,6$ & $7,2 \mathrm{a}$ \\
\hline 9 & $2,8 \pm 0,4$ & $3,2 \pm 0,8$ & $6,5 \pm 0,8$ & $3,5 \pm 0,3$ & $2,5 \pm 0,1$ & $2,4 \pm 0,3$ & $3,5 \mathrm{~d}$ \\
\hline Surubim-41 & $6,9 \pm 0,7$ & $4,9 \pm 0,4$ & $6,0 \pm 0,6$ & $4,6 \pm 1,2$ & $3,1 \pm 0,1$ & $1,8 \pm 0,3$ & $4,6 \mathrm{c}$ \\
\hline IAC 12.829 & $4,9 \pm 0,2$ & $3,0 \pm 0,3$ & $5,7 \pm 0,9$ & $3,3 \pm 0,5$ & $5,7 \pm 0,0$ & $5,6 \pm 0,1$ & $4,7 \mathrm{c}$ \\
\hline 1468 & $6,4 \pm 0,1$ & $5,8 \pm 0,4$ & $5,5 \pm 0,1$ & $3,5 \pm 0,3$ & $2,4 \pm 0,5$ & $2,4 \pm 0,0$ & $4,3 \mathrm{c}$ \\
\hline Amarrilla & $3,7 \pm 0,3$ & $3,6 \pm 0,5$ & $5,4 \pm 1,4$ & $3,8 \pm 0,2$ & $2,3 \pm 0,1$ & $2,1 \pm 0,4$ & $3,5 \mathrm{~d}$ \\
\hline CM 3360-4 & $3,6 \pm 0,2$ & $2,9 \pm 0,3$ & $3,1 \pm 0,2$ & $2,0 \pm 1,0$ & $3,1 \pm 0,1$ & $2,7 \pm 0,2$ & 2,9 ed \\
\hline 60 & $4,2 \pm 1,2$ & $3,9 \pm 1,0$ & $4,5 \pm 0,7$ & $3,1 \pm 0,3$ & $2,6 \pm 0,6$ & $2,3 \pm 0,1$ & $3,4 d$ \\
\hline Catiguá & $4,5 \pm 0,7$ & $3,0 \pm 0,4$ & $2,4 \pm 0,6$ & $2,7 \pm 0,8$ & $2,5 \pm 0,3$ & $1,0 \pm 0,2$ & $2,7 \mathrm{e}$ \\
\hline M Par 75 & $4,1 \pm 0,4$ & $2,8 \pm 0,6$ & $2,1 \pm 0,4$ & $1,8 \pm 0,6$ & $2,8 \pm 0,3$ & $2,4 \pm 0,2$ & $2,7 \mathrm{e}$ \\
\hline EC 55 & $3,6 \pm 0,3$ & $3,0 \pm 0,4$ & $2,6 \pm 0,8$ & $2,1 \pm 0,3$ & $1,5 \pm 0,1$ & $1,2 \pm 0,1$ & $2,3 \mathrm{fe}$ \\
\hline 76 & $2,0 \pm 0,3$ & $1,4 \pm 0,3$ & $2,0 \pm 0,3$ & $1,6 \pm 0,3$ & $1,5 \pm 0,3$ & $1,0 \pm 0,2$ & $1,6 \mathrm{hg}$ \\
\hline Carapé & $2,7 \pm 0,1$ & $1,9 \pm 0,3$ & $1,7 \pm 0,3$ & $0,9 \pm 0,1$ & $0,3 \pm 0,1$ & $0,4 \pm 0,1$ & $1,3 \mathrm{hg}$ \\
\hline EC 11 & $2,3 \pm 0,5$ & $1,7 \pm 0,4$ & $1,4 \pm 0,1$ & $1,4 \pm 0,1$ & $1,0 \pm 0,1$ & $1,0 \pm 0,1$ & $1,5 \mathrm{hg}$ \\
\hline EC 27 & $3,5 \pm 0,3$ & $3,1 \pm 0,1$ & $3,2 \pm 0,2$ & $2,1 \pm 0,2$ & $2,3 \pm 0,1$ & $1,8 \pm 0,2$ & $2,7 \mathrm{e}$ \\
\hline EC 118 & $2,4 \pm 0,5$ & $2,0 \pm 0,1$ & $2,7 \pm 0,4$ & $1,8 \pm 0,6$ & $1,6 \pm 0,1$ & $0,9 \pm 0,3$ & $1,9 \mathrm{gf}$ \\
\hline EC 64 & $2,0 \pm 0,0$ & $1,1 \pm 0,1$ & $1,3 \pm 0,4$ & $1,0 \pm 0,2$ & $0,9 \pm 0,0$ & $0,9 \pm 0,1$ & $1,2 \mathrm{~h}$ \\
\hline EC 78 & $2,2 \pm 0,3$ & $1,0 \pm 0,1$ & $1,3 \pm 0,3$ & $0,9 \pm 0,1$ & $0,4 \pm 0,1$ & $0,8 \pm 0,1$ & $1,1 \mathrm{~h}$ \\
\hline $\begin{array}{l}\text { Media por } \\
\text { subcultivo }\end{array}$ & $3,9 \mathrm{a}$ & $3,4 \mathrm{~b}$ & $4,0 \mathrm{a}$ & $3,0 \mathrm{c}$ & $2,9 \mathrm{c}$ & $2,3 d$ & $3,3 \pm 0,3$ \\
\hline CV (\%) & 39,4 & 53,5 & 57,7 & 63,4 & 77,4 & 76,6 & 53,4 \\
\hline
\end{tabular}

${ }^{* 1}$ Media aritmética \pm error estándar.

*2 Letras distintas indican diferencias significativas según el test de comparaciones múltiples de Duncan $(\mathrm{P} \leq 0,05)$.

forma, tamaño y coloración. Consecuentemente no fueron identificadas variantes que sean morfológicamente reconocibles como fuera de tipo. Según Roca (1984) la formación de callos en sistemas de micropropagación es indeseable por promover la ocurrencia de variantes somaclonales, siempre y cuando comprenda el tejido del cual se regeneran las plantas ya sea por vía organogénica o embriogénica. En el presente trabajo, se observó formación de callos en algunos explantes de todos los cultivares evaluados; sin embargo estos callos se diferenciaron apenas en la base del segmento nodal y fueron de aspecto compacto, de coloración blanco amarillenta, no presentando capacidad caulogénica ni embriogénica, ni obstaculizando la brotación normal de la yema lateral del segmento nodal empleado como explante, garantizando así la estabilidad genética del material multiplicado.
En otras especies multiplicadas in vitro mediante organogénesis directa se ha demostrado la estabilidad genética de su progenie sexual como en arroz (Medina et al., 2004) o de su progenie clonal como en Solanum aculeatissimum (Ghimire et al., 2012) y Chlorophytum borivilianum (Basu y Jha, 2014). Por el contrario, se han detectado variantes somaclonales de Lippia integrifolia en vástagos regenerados por organogénesis directa a partir del cultivo in vitro de meristemas axilares y vástagos regenerados de novo a partir de callos organogénicos, mediante el análisis de marcadores moleculares basados en microsatélites anclados (Iannicelli et al., 2016), por lo que se hace indispensable considerar la evaluación de la estabilidad genética de todas las plantas recuperadas, independientemente de su vía de regeneración.

Conocer la tasa de multiplicación in vitro de 


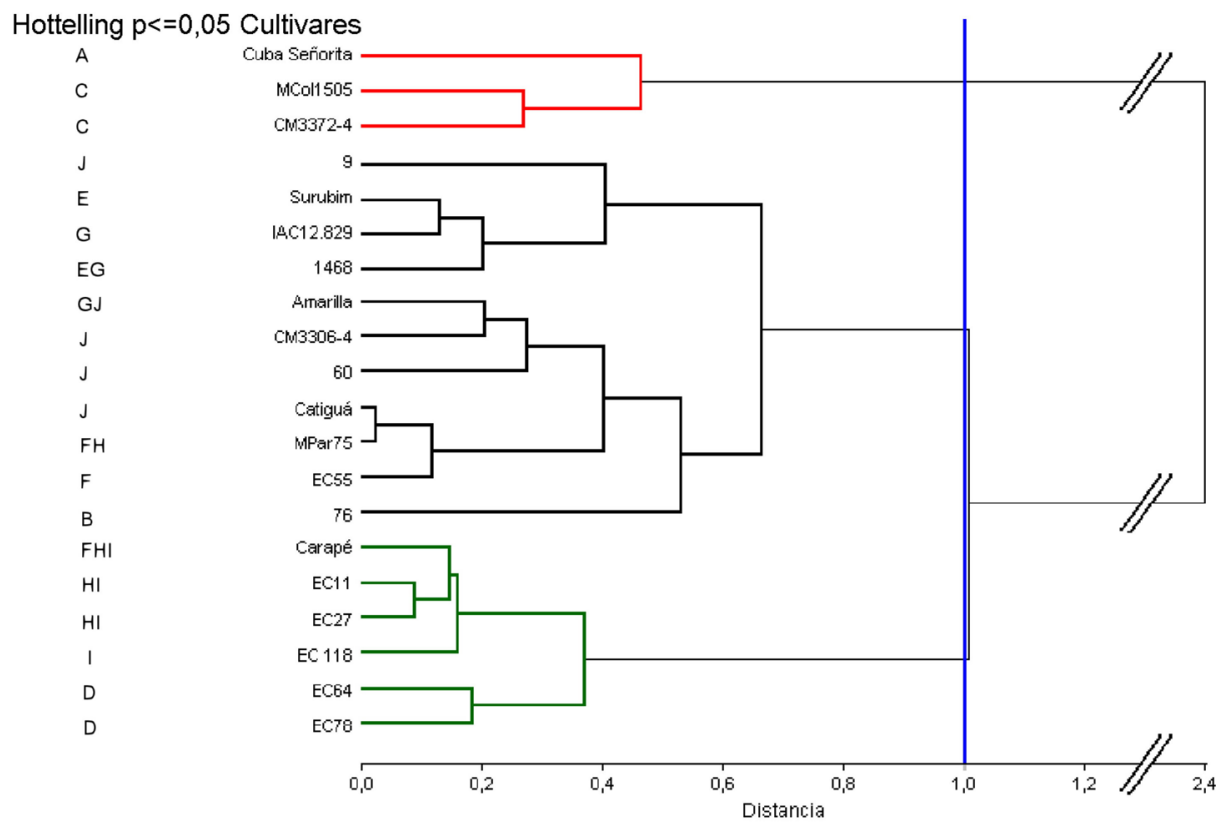

Fig. 2. Fenograma de 20 cultivares de mandioca empleando el método del promedio aritmético de grupos de pares no ponderados (UPGMA) y la distancia Euclidiana promedio de 3 variables cuantitativas (número promedio de nudos regenerados por explante por subcultivo, número promedio de nudos acumulados totales y longitud promedio de los vástagos regenerados por explante por subcultivo). Coeficiente Correlación Cofenética $=0,731$.

un determinado cultivar de mandioca así como su comportamiento en relación al crecimiento es importante cuando se desea llevar adelante un programa de propagación masal (Pedroso de Oliveira et al., 2000). Ambas características pueden servir como herramienta para la toma de decisiones al momento de proyectar la producción de un número determinado de plantas de materiales selectos, la realización de nuevos subcultivos o el pasaje a tierra para la readquisición de vigor para iniciar nuevos establecimientos in vitro. La eficiencia del procedimiento empleado se optimizará si se iniciara la micropropagación con plantas libres de virus derivadas del cultivo de meristemas caulinares (Schaller et al., 2014).

\section{ConCLusión}

En resumen, el protocolo de micropropagación empleado en este trabajo brinda la posibilidad de regenerar plantas in vitro de 20 cultivares durante 180 días (6 subcultivos totales realizados cada
30 días) corroborando un efecto pronunciado del genotipo sobre la eficiencia de la micropropagación de mandioca. Se identificó variabilidad debida al genotipo, distinguiéndose 3 grupos de cultivares de acuerdo al número promedio de nudos acumulados por subcultivo, número promedio de nudos acumulados totales y la longitud de los vástagos regenerados. El agrupamiento de cultivares también brindará información valiosa para el ajuste de procedimientos que permitan optimizar la eficiencia de la micropropagción de mandioca para establecer un programa de propagación masal sencillo y exitoso.

\section{Agradecimientos}

A la Agencia Nacional de Promoción Científica y Tecnológica (PICT 2309-2012), la Secretaría General de Ciencia y Técnica de la Universidad Nacional del Nordeste (PI A002/11 y PI A00/14) y el Consejo Nacional de Investigaciones Científicas y Técnicas (PIP Nº11220150100398 CO) por financiar este trabajo. 


\section{Biblografía}

ACEDO, V. 1994. Meristem culture and in vitro maintenance of Philippine cassava. In: Proceedings of the Second International Scientific Meeting on Cassava Biotechnology Network, pp.202209. Cassava Biotechnology Network, Centro Internacional de Agricultura Tropical (CIAT), Bogor.

ACEDO, V. 2006. Improvement of in vitro techniques for rapid meristem development and mass propagation of Philippine cassava (Manihot esculenta Crantz). J. Food, Agri. Environ. 4: 220-224.

ALBARRÁN J., F. FUENMAYOR \& M. FUCHS. 2003. Propagación clonal rápida de variedades comerciales de yuca mediante técnicas biotecnológicas. Revista Digital CENIAP Hoy $\mathrm{N}^{\circ} 3$.

BASU, S. \& T.B. JHA. 2014. Direct organogenesis, phytochemical screening and assessment of genetic stability in clonally raised Chlorophytum borivilianum. Environ. Exp. Biol. 12: 167-178

BROOMES, V.F. \& R. LACON. 1994. Influence of medium components on hardening of cassava after micropropagation in liquid nutrient medium. In: Proceedings of the Second International Scientific Meeting on Cassava Biotechnology Network, pp.210-219. Cassava Biotechnology Network, CIAT, Bogor.

CAVALLERO, M., R. MEDINA (EX AEQUO), R. HOYOS, P. CENÓZ, \& L. MROGINSKI. 2012. Biotechnology applied to cassava propagation in Argentina. In: PACE, C. (ed.), Cassava: Farming, Uses, and Economic Impact, pp. 55-77. Nova Science Publishers, USA.

CEBALLOS, H. \& G. DE LA CRUZ. 2002. Taxonomía y. Morfología de la yuca. En: OSPINA, B. \& H. CEBALLOS (eds.), La yuca en el tercer milenio: sistemas modernos de producción, procesamiento, utilización y comercialización, pp. 16-32. CIAT, Cali, Colombia.

COCK, J. 1985. Cassava: new potential for a neglected crop. Westview Press, Boulder and London.

EL-SHARKAWY, M. 2003. Cassava biology and physiology. Plant Mol. Biol. 53: 621-641.

FREGENE, M., J. TOHME, W. ROCA, P. CHAVARRIAGA, R. ESCOBAR \& H. CEBALLOS. 2002. Biotecnología de yuca. En: OSPINA B. \& H. CEBALLOS (eds.), La yuca en el tercer milenio: sistemas modernos de producción, procesamiento, utilización y comercialización, pp. 377-405. CIAT, Cali, Colombia.

GHIMIRE, B.K., C.Y. YU \& I.M. CHUNG. 2012. Direct shoot organogenesis and assessment of genetic stability in regenerants of Solanum aculeatissimum Jacq. Plant Cell Tiss. Org. Cult. 108: 455-464.
HANKOUA, B., S. NG, I. FAWOLE, J. PUONTIKAERLAS, M. PILLAY \& A. DIXON. 2005. Regeneration of a wide range of African cassava genotypes via shoot organogenesis from cotyledons of maturing somatic embryos and conformity of the field-established regenerants. Plant Cell Tiss. Org. Cult. 82: 221-231.

IANNICELLI, J., M. PÉREZ DE LA TORRE, A. COVIELla, E. DEL VALLE AGUiRRE, M.A. ELECHOSA, C.M. VAN BAREN, M.G. PACHECO \& A.S. ESCANDÓN. 2016. In vitro propagation of Lippia integrifolia (Griseb.) Hier. and detection of genetic instability through ISSR markers of in vitro-cultured plants. Rev. Fac. Agron. 115: 67-76

DEMEKE, Y., W. TEFERA, N. DECHASSA \& B. ABEBLE. 2014. Effects of plant growth regulators on in vitro cultured nodal explants of cassava (Manihot esculenta Crantz) clones. Afr. J. Biotechnol. 13: 2830-2839.

DI RIENZO, J.A., F. CASANOVES, M.G. BALZARINI, L. GONZALEZ, M. TABLADA \& C.W. ROBLEDO. 2008. InfoStat versión 2008, Grupo InfoStat, FCA, Universidad Nacional de Córdoba, Argentina.

LÓPEZ, J. 2002. Semilla vegetativa de yuca. En: OSPINA, B. \& H. CEBALLOS (eds.), La yuca en el tercer milenio: sistemas modernos de producción, procesamiento, utilización y comercialización, pp. 49-75, CIAT, Cali, Colombia.

MEDINA, R. D., M.M. FALOCI, M.A. MARASSI \& L.A. MROGINSKI. 2004. Genetic stability in rice micropropagation. Biocell 28: 13-20.

MURASHIGE, T. \& F. SKOOG. 1962. A revised medium for rapid growth and bioassay with tobacco tissue cultures. Physiol. Plant. 15: 473-497.

MUSSIO, I., M. CHAPUT, I. SERRAF, G. DUCREUX \& D. SIHACHAKR. 1998. Adventitious shoot regeneration from leaf explants of an African clone of cassava (Manihot esculenta Crantz) and analysis of the conformity of regenerated plants. Plant Cell Tiss. Org. Cult. 53: 205-211.

PEDROSO DE OLIVEIRA, R., T. DA SILVA GOMES \& A. DUARTE VILARINHOS. 2000. Avaliação de um sistema de micropropagação massal de variedades de mandioca. Pes. Agrop. Bras. 35: 2329-2334.

PUONTI-KAERLAS, J. 1998. Cassava Biotechnology. Biotechnol. Genet. Eng. Rev. 15: 329-364.

ROCA, W.M. 1984. Cassava. En: SHARP, W.R., D.A. EVANS, P.V. AMMIRATO \& Y. YAMADA (eds.), Handbook of plant cell culture: crop species, pp. 269-301. Macmillan Publishing Co., New York.

ROCA, W., B. NOLT, G. MAFLA, J. ROA \& R. REYES. 1991. Eliminación de virus y propagación de clones 
R. D. Medina et al. - Eficiencia de la micropropagación de genotipos de mandioca

en la yuca (Manihot esculenta Crantz). En: W. ROCA \& L. MROGINSKI (eds.), Cultivo de tejidos en la agricultura: fundamentos y aplicaciones, pp. 403-420. CIAT, Cali, Colombia.

SCHALLER, S.C., A. ZANINI, P. RODRÍGUEZ PARDINA, L. DI FEO, L. MROGINSKI \& R.D. MEDINA. 2014. Regeneración de plantas de mandioca por cultivo de meristemas para su empleo en estudios de virología en la Argentina. En: L.D. PLOPLER (Ed.), Proceedings of the III Congreso Argentino de Fitopatología, pp. 436. Asociación Argentina de Fitopatólogos, San Miguel de Tucumán, Argentina.
SMITH M. K., B. J. BIGGS \& K. J. SCOTT. 1986. In vitro propagation of cassava (Manihot esculenta Crantz). Plant Cell Tiss. Org. Cult. 6: 221-228.

Recibido el 1 de septiembre de 2016, aceptado el 8 de noviembre de 2016. 
